\title{
Prediction of pressure due to elastic fabric tube following energy principle
}

\begin{abstract}
Elastic knitted fabric tubes are used as pressure garments and pressure bandages in compression therapy to mitigate many physical ailments of human beings. Laplace law has been extensively used for prediction of pressure at the interface of human body and elastic tube by many. However, it has been stated to be inadequate in some cases. Human body is compressible because of presence of soft tissues. This characteristic has not been taken into account. In the present paper a mathematical model based on energy principle has been proposed to predict pressure by a fabric tube fitted on foam covered rigid cylinder. The model equation has shown better prediction of the interfacial pressure than that one expects based on Laplace's law.
\end{abstract}

Volume 2 Issue 5 - 2017

\author{
R Chattopadhyay, Moumita Bera \\ Department of Textile Technology, India
}

Correspondence: R Chattopadhyay, Department of Textile Technology, IIT Delhi, Hauz Khas, New Delhi, India, Tel 9871095892, Email rabisankarc@gmail.com

Received: July 09, 2017 | Published: August 24, 2017

\section{Introduction}

Pressure garments as well as pressure bandages are extensively used in compression therapy. Pressure garments are elastic knitted fabric tubes and pressure bandages are stretchable narrow fabrics. Pressure garments can be directly worn whereas; pressure bandages are to be wrapped under uniform tension around a limb. Tension in the curved shape bandage or garment as they follow the body contour is the main source of radial pressure. The compression exerted by pressure garments therefore primarily depends upon factors, such as elastic modulus of the fabric, the stretch in it (reduction factor of the garment), curvature of the body and compliance of the body part on which the garment is worn. Elastic knitted fabrics, used in pressure garments, are either warp or weft knitted fabric with inlaid elastic yarns. The garment circumference is shorter than the limb circumference and the percentage change in the difference in circumference is known as reduction factor. The tension in a fabric at a given reduction factor would change with change in intrinsic property of the elastic inlay yarn, its thickness (denier) and numbers per unit width.

It is extremely important that the fabric tube exert desired pressure on the tissues. To ensure right pressure one needs to engineer tensile characteristics of the fabric tube keeping in mind the body shape and size. A proper understanding of the relationship between the property of the fabric, body characteristics, its dimensions and pressure generation is necessary.

The interface pressure with and without the use of a custom fitted pressure garments have been shown to be less at the calf (muscular area) than at the ankle (bony area). ${ }^{1}$ The expected pressure estimated on the basis of Laplace law has been reported to be inadequate in some cases..$^{2-4}$ It appears that compressibility of the body parts and curvature must have a significant effect.

Many parts of the human body are compressible. This characteristics needs to be taken into account for estimating pressure. In the present paper a mathematical model based on energy principle has been proposed to predict pressure generated by an elastic fabric tube fitted on foam covered rigid PVC cylinder.

\section{Principle of pressure development}

To exert pressure on any part of the human body either a flat fabric or an elastic knitted tube are used. In the case of fabric, one needs to wrap the body by it under tension and for elastic tube, a tube smaller than the body part, is worn so that the fabric remains under tension. The tension generates inward radial force as it passes over the curved body part and thereby compresses the limb. Therefore, curvature and stretch are necessary conditions for development of radial pressure.

Many researchers have tried to associate pressure with tension and curvature. According to Laplace's law, the pressure gradient across a closed elastic spherical membrane and the corresponding tension generated in it are directly proportional as shown below: ${ }^{5-6}$

$$
\left(P_{\alpha}-P_{\beta}\right)=\frac{2 T_{e}}{r}
$$

Where, $P_{\alpha}$ and $P_{\beta}$ are the internal and external pressures on the spherical surface respectively, $T_{e}$ is the tension generated in the sphere wall and $r$ is the radius of curvature of the body in. The factor 2 on the right hand side appears as the membrane experiences biaxial stresses being spherical.

When a cylinder with closed ends is subjected to internal pressure $(\mathrm{P})$, the tension in the wall of it has been shown to be: ${ }^{3}$

$$
P=\frac{T_{e}}{r}
$$

Many researchers have proposed modified forms of Laplace's Law to predict the interfacial pressure between garment and human body. To find out the pressure at knee position, Kirk \& Ibrahim $^{7}$ reported the following relationship:

$$
P=\frac{T_{H}}{\rho_{H}}+\frac{T_{V}}{\rho_{V}}
$$

Where, $P$ denotes pressure, $T_{H}$ is the tensile stress in horizontal direction in, $T_{V}$ represents the tensile stress in vertical direction, $\rho_{H}$ 
refers to radius of curvature in horizontal direction in inch and $\rho_{V}$ is the radius of curvature in vertical direction.

Seo et al. ${ }^{8}$ introduced fabric thickness $\left(h_{l}\right)$ in Equation 4 and proposed the following relationship:

$$
P=\frac{\sigma_{H} \times h_{1}}{r_{H}}+\frac{\sigma_{V} \times h_{1}}{r_{V}}
$$

Where, $\sigma_{H} \& \sigma_{V}$ denote tensile stresses and $r_{H}$ and $r_{V}$ radius of curvatures in the horizontal and cross directions respectively.

Continuing the modification of the Laplace's Law, Lee ${ }^{9}$ proposed the 'principal stress' and 'principal directions' instead of the two orthogonal directions. The tensile stresses and radii of curvature were measured along the maximum and minimum principal directions. The model to calculate the pressure was:

$$
P=\frac{\sigma P \max \times t}{r P \max }+\frac{\sigma P \min \times t}{r P \min }
$$

Where, $\sigma P \max$ refers to tensile force per unit area in maximum principal direction $\sigma P$ min denotes tensile force per unit area in minimum principal direction and, $r_{P} \max$ and $r_{P}$ min indicates the radius in horizontal and vertical directions, respectively and $t$ is the thickness.

Hui \& $\mathrm{Ng}^{10}$ proposed the following equation to predict the pressure exerted by multilayered fabric by taking into account the thickness of each layer.

$$
P=\frac{e 2 \pi \sum_{i=1}^{N}\left(E_{i} h_{i}\right)}{C}
$$

The equation demonstrates that, the final pressure exerted by a multilayered fabric tube is dependent on the elastic modulus $(E)$ of the fabric, thickness $(h)$ of each layer, number of layers $(N)$, total strain $(e)$ and circumference of the body $(C)$.

Several studies validated the Laplace's equation for predicting pressure on a rigid body. However, according to Seo H et al. ${ }^{8}$ Lee YJ, ${ }^{9}$ Hui CL \& Ng SF, ${ }^{10}$ the predicted pressure on human body which is compress-able, was not very good. This was attributed to the difficulty in measuring the radius of curvature of a human limb which is not perfectly circular. To overcome this limitation, Macintyre ${ }^{11}$ modified the equation and introduced circumference of the body part instead of radius of curvature in the equation (Equation 8). The author also changed the units of pressure from Pascal to $\mathrm{mmHg}$ (unit used for measuring capillary pressure) and found to be more suitable for estimating the pressure (in $\mathrm{mmHg}$ ) on human body.

$$
P=\frac{4.713 \times T_{1}}{C}
$$

Where, $T_{1}$ is the tension generated per unit width of fabric in textile and $C$ refers to the limb circumference. In case of bandages, Yildiz ${ }^{12}$ brought fabric width $(W)$ in the equation:

$$
P=\frac{4620 \times T_{e}}{C \times W}
$$

One can observe that all these models are essentially based on Laplace's basic Equation (1) which is based on the principle of force equilibrium. Recently Lihuan Zhao et al..$^{13}$ have made an excellent review of different formulae proposed by many researchers.

\section{Pressure estimation based on energy principle}

As stated earlier the source of pressure is fabric extension and curvature of body on which it is worn. In case of human limb, as soon as the stretched fabric tube is placed on any part of the limb and released, the soft tissues gets compressed and the diameter of the corresponding portion of the limb reduces. This is so as part of the stored elastic energy of the fabric is spent in compressing the tissues.

A similar situation is expected if the fabric tube is placed over a foam covered rigid cylinder simulating human body consisting of bone \& tissues. At equilibrium, Energy released by the fabric tube $=$ Compressive energy required to deform the foam.

By knowing the energy released by the fabric, the pressure acting on the foam can be determined, if the pressure-compression characteristics of the foam and force-elongation characteristics of the fabric tube are known.

Figure 1 shows a schematic of PVC foam covered cylinder before and after deformation by the elastic fabric tube. Let,

$D_{1}=$ diameter of the rigid cylinder

$D_{2}=$ diameter of the foam covered cylinder

$D_{3}=$ diameter of foam covered cylinder in compressed state

$h_{0}=$ initial thickness of foam

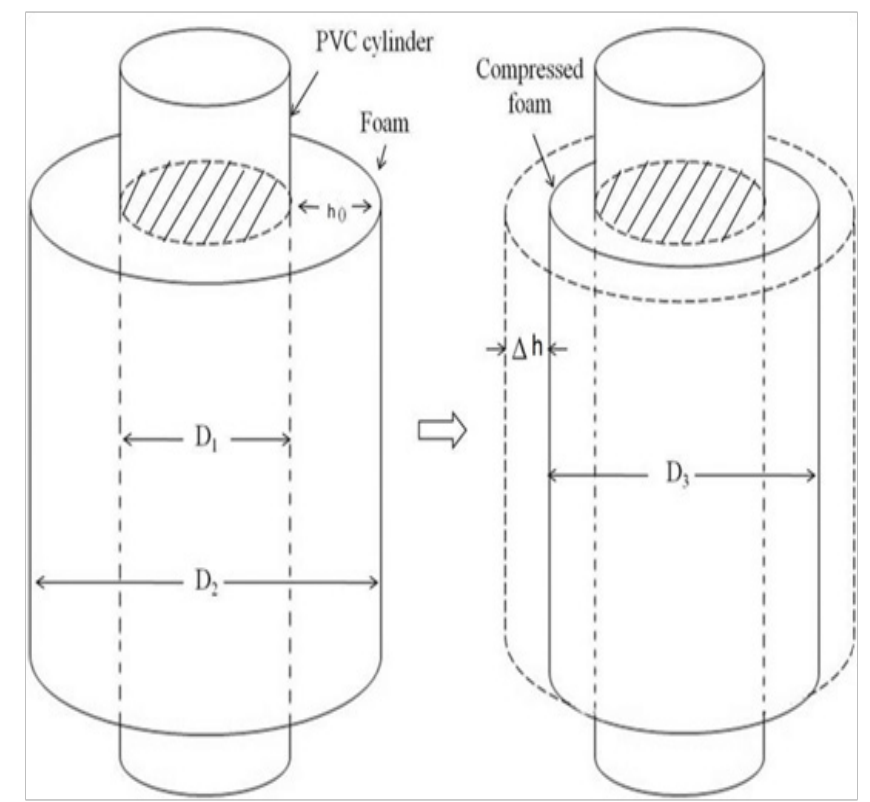

Figure I Schematic diagram of foam covered PVC cylinder.

As soon as the fabric is placed on the PVC cylinder, the foam gets compressed and the diameter reduces from $D_{2}$ to $D_{3}$. Considering, $\Delta h$ as the reduction in thickness of the foam, $D_{2}$ and $D_{3}$ can be written as

$$
D_{2}=D_{1}+2 h_{0}
$$




$$
D_{3}=D_{2}-2 \Delta h
$$

Determination of Fabric stretch: Let, The circumference of the fabric tube in relaxed state $=l_{0}$. Circumference of the foam covered cylinder (before deformation) $=\pi D_{2}$

Initial elongation $\left(\varepsilon_{0}\right)$ of the fabric tube (as it is mounted on the cylinder): $\varepsilon_{0}=\pi D_{2}-l_{0}$. The diameter of foam covered cylinder reduces from $D_{2}$ to $D_{3}$ due to contraction of the fabric. Therefore at equilibrium state, the elongation $\left(\varepsilon_{l}\right)$ of the fabric would reduce from

$\varepsilon_{0}$ to $\varepsilon_{1}$ i.e. $\varepsilon_{1}=\pi D_{3}-l_{0}$

The load elongation curve of a fabric tube is shown in Figure 2. Assume that the curve remains same during loading and unloading phases. Let, the following polynomial describes the load- elongation relationship of the fabric tube:

$$
F=f(\varepsilon)=a \varepsilon^{2}+b \varepsilon+c
$$

Where, $F$ is the force at any elongation $\varepsilon$, and $a, b$ and $c$ are the coefficients of the polynomial. Therefore, the energy released ( $E_{F a b}$ ) by the fabric while, its elongation changes from $\varepsilon_{0}$ to $\varepsilon_{1}$ can be given by Equation 12 .

$$
E_{F a b}=-\int_{\varepsilon_{0}}^{\varepsilon_{1}}\left(a \varepsilon^{2}+b \varepsilon+c\right) d \varepsilon \quad E_{F a b}=\int_{\varepsilon_{1}}^{\varepsilon_{0}}\left(a \varepsilon^{2}+b \varepsilon+c\right) d \varepsilon
$$

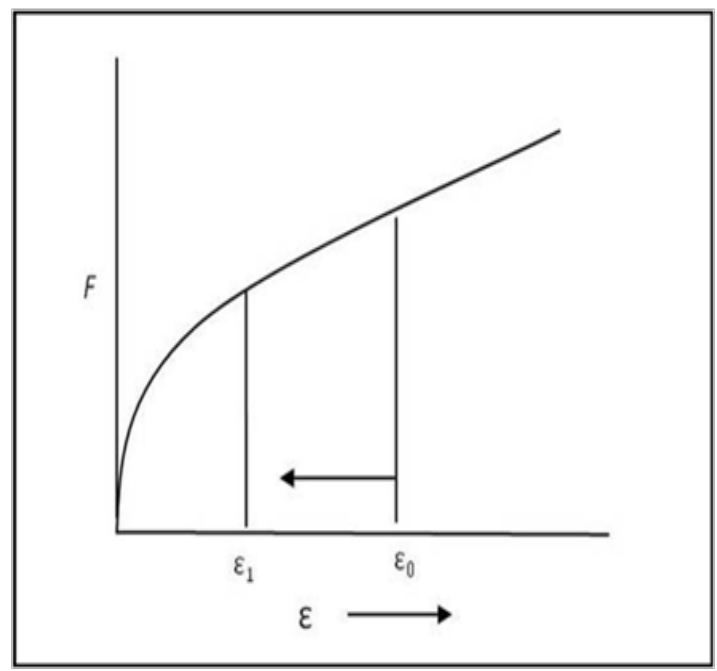

Figure 2 Typical load - elongation curve of fabric.

A typical thickness-pressure curve of foam shown in Figure 3 can be represented by the following polynomial:

$$
h=f(p)=\alpha p^{2}+\beta p+\gamma
$$

Where, $h$ denotes the thickness of the foam at pressure $p$, and $\alpha, \beta$ $\& \gamma$ are the coefficients of the polynomial.

Let, the initial thickness of the foam at $p=0$ be $h_{0}$. Hence, $h_{0}=\gamma$ The change in thickness of the foam at any pressure $p$ would be

$$
-\Delta h=h_{0}-h=\alpha p^{2}+\beta p
$$

Therefore, the compressive energy ( $E_{\text {Foam }}$ ) required for deforming the foam, as pressure increases from 0 to $p$ can be expressed as:

$E_{\text {Foam }}=-\int_{h_{0}}^{h} p d h=-\int_{0}^{p} p(2 \alpha p+\beta) d p=-\int_{0}^{p}\left(2 \alpha p^{2}+\beta p\right) d p=-\left[2 \alpha \frac{p^{3}}{3}+\beta \frac{p^{2}}{2}\right]$

Since, $E_{\text {Fab }}=E_{\text {foam }}$

$$
\therefore \int_{\varepsilon_{1}}^{\varepsilon_{0}}\left(a \varepsilon^{2}+b \varepsilon+c\right) d \varepsilon=-\left[2 \alpha \frac{p^{3}}{3}+\beta \frac{p^{2}}{2}\right]
$$

By determining the constants of the polynomial describing the load elongation relationship of fabric tube on the basis of the experimental data, one can find out the energy released by the fabric as its elongation reduces from $\varepsilon_{0}$ to $\varepsilon_{\text {. }}$. Similarly, the coefficients $\alpha$ and $\beta$ can be found out from the pressure-deformation data of the foam. By equating known values of the energy released by a given fabric, the unknown pressure $p$ on foam can be estimated.

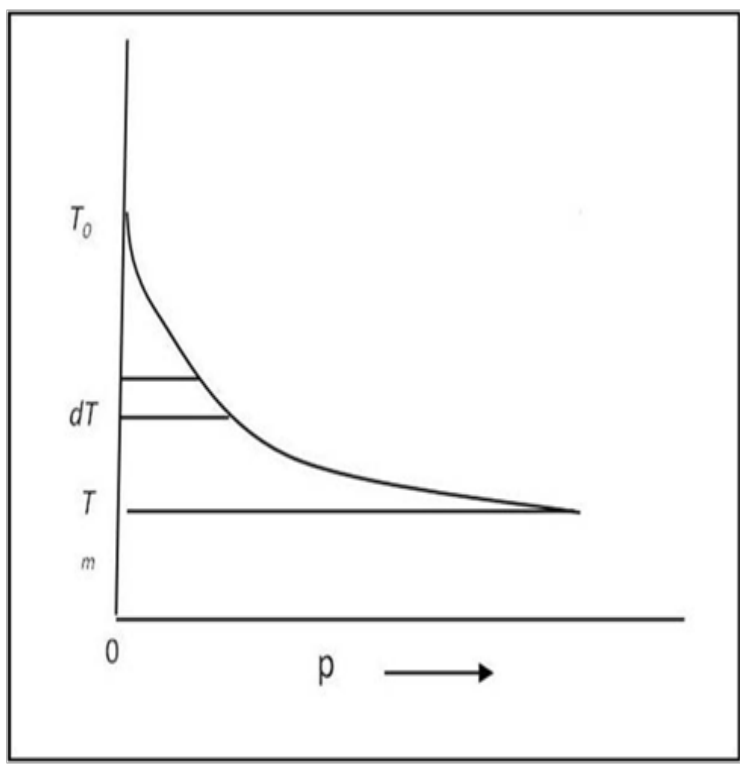

Figure 3 Typical compression deformation curve of foam.

\section{Experimental}

\section{Material}

In order to simulate the human body consisting of soft tissues over bones PVC cylinder and foam were used. Two rigid PVC cylinders of radii $3.3 \mathrm{~cm}$ and $4.6 \mathrm{~cm}$ were chosen. The height and wall thickness of the cylinders were $20 \mathrm{~cm}$ and $2 \mathrm{~mm}$ respectively. Piece of foam was attached on the outer surface of the smaller cylinder by an adhesive so that the radius of the cylinder with foam becomes almost equal to $4.6 \mathrm{~cm}$.

Knitted fabric tubes were prepared in single jersey construction on a circular knitting machine with a cylinder diameter of $9.52 \mathrm{~cm}$ and 
needle gauge of $6.69 \mathrm{per} \mathrm{cm}$. The yarns used were: a combination of 210 denier textured nylon and nylon covered 20 denier spandex yarns for loop formation and nylon covered 420 denier spandex filaments as inlay. The fabric sample was tested on Instron tensile tester for determining the force - elongation relationship.

An empirical equation that describes the load elongation graph of the chosen fabric tube (Figure 4) was found to be:

$$
F=-0.0002 \varepsilon^{2}+0.0301 \varepsilon+0.121
$$

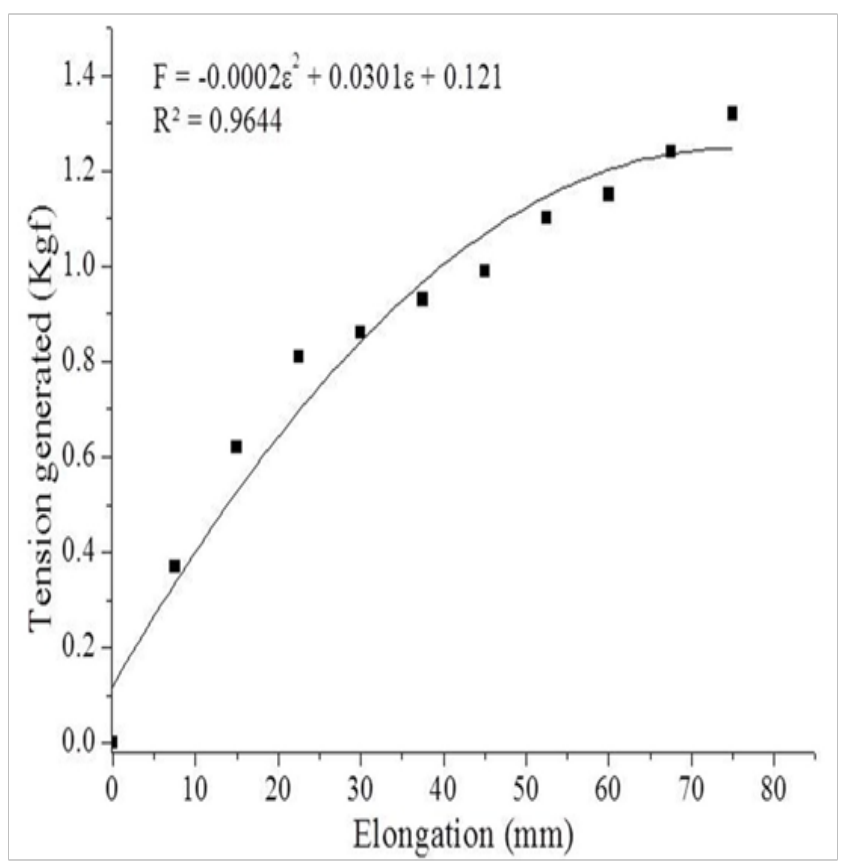

Figure 4 Actual Load elongation behaviour of fabric.

\section{Foam characterization}

The compression characteristic of the foam was determined by using Essdiel (Manchester, UK) thickness tester. The area of the compression probe of the instrument was $3.5 \mathrm{~cm}^{2}$. The foam thickness was measured keeping initial pressure at $20 \mathrm{gf} / \mathrm{cm}^{2}$ and its thickness was found to be $11.08 \mathrm{~mm}$. The applied pressure was gradually increased to $50 \mathrm{gf} / \mathrm{cm}^{2}, 100 \mathrm{gf} / \mathrm{cm}^{2}, 200 \mathrm{gf} / \mathrm{cm}^{2}, 500 \mathrm{gf} / \mathrm{cm}^{2}, 1000 \mathrm{gf} / \mathrm{cm}^{2}$, and $1500 \mathrm{gf} / \mathrm{cm}^{2}, 2000 \mathrm{gf} / \mathrm{cm}^{2}$ by adding weights on the probe i.e. disc in steps and the thickness values were noted down. Thereafter, the dead weights were removed one by one and thickness readings were further noted. A delay of at least 60 seconds was maintained between two load applications. A minimum of five observations were made and average thickness was calculated.

The equation that best describes the compressive force-deformation graph (Figure 5) of the foam used in the study is:

$$
\Delta h=-2 \times 10^{-7} p^{2}+0.0008 p
$$

Hence, $\alpha$ and $\beta$ of equation 19 are $-2 \times 10^{-7}$ and 0.0008 respectively.

\section{Pressure measurement}

The fabric tube diameter was suitably adjusted so that the pressure could be measured at four different reduction factors, namely, $10 \%$, $20 \%, 30 \%$, and $40 \%$, (Table 1 ). Reduction factor is the $\%$ by which the fabric tube circumference is shorter than PVC cylinder circumference. After placing the fabric tube on the cylinder, the pressure at the interface of the tube and fabric was measured by Kikuhime pressure sensor. While measuring pressure, the sensor was adjusted to show a zero reading on the pressure display monitor. The balloon probe was fixed on the soft and rigid PVC cylinders with a cello tape. The fabric tube was then carefully mounted on the cylinder so that, no displacement or distortion of the sensor occurs. Thereafter, the fabric tube was adjusted so that it was uniformly spread out across the cylinder circumference. The fabric tube was then, allowed to settle and relax in position for 30 seconds before pressure reading was recorded. At least 5 readings were taken and an average was calculated.

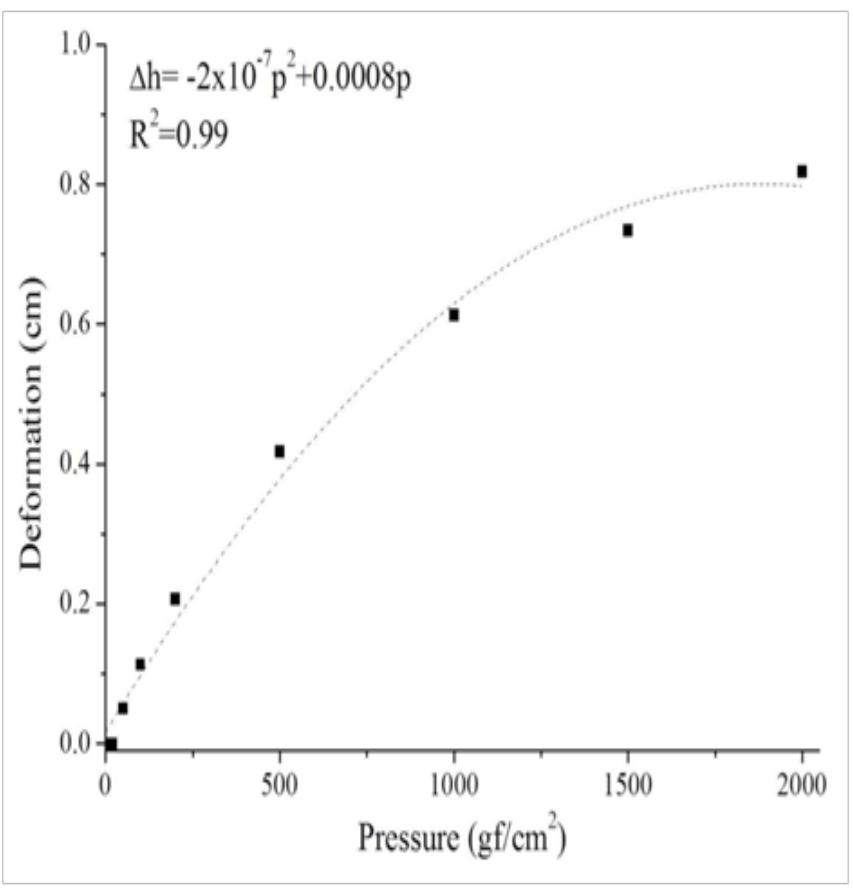

Figure 5 Deformation vs. pressure relationship of foam.

\begin{tabular}{|c|c|c|c|c|c|}
\hline $\begin{array}{l}\text { S } \\
\text { No }\end{array}$ & $\begin{array}{l}\text { Bare } \\
\text { PVC } \\
\text { cylinder } \\
\text { radius } \\
(\mathrm{cm})\end{array}$ & $\begin{array}{l}\text { Cylinder } \\
\text { type and } \\
\text { radius } \\
(\mathrm{cm})\end{array}$ & $\begin{array}{l}\text { Curvature } \\
(1 / \mathrm{cm})\end{array}$ & $\begin{array}{l}\text { Reduction } \\
\text { factor }(\%)\end{array}$ & $\begin{array}{l}\text { Fabric } \\
\text { tube } \\
\text { radius } \\
(\mathrm{cm})\end{array}$ \\
\hline I & & Soft & & 10 & 3.9 \\
\hline 2 & 3.3 & & 0.23 & 20 & 3.5 \\
\hline 3 & & 4.4 & & 30 & 3.1 \\
\hline 4 & & & & 40 & 2.6 \\
\hline 5 & & Rigid & & 10 & 4.1 \\
\hline 6 & 4.6 & & 0.22 & 20 & 3.7 \\
\hline 7 & & 4.6 & & 30 & 3.2 \\
\hline 8 & & & & 40 & 2.8 \\
\hline
\end{tabular}

Table I Dimensions of PVC cylinders 


\section{Results and discussion}

\section{Comparison between measured pressure and estima- ted by laplace law}

Figure 6 depicts the reduction factor Vs pressure graphs on rigid and foam covered PVC cylinders. The pressure varied from 0 to $35 \mathrm{mmHg}$ for rigid cylinder and from 0 to $33 \mathrm{mmHg}$ for foam covered PVC cylinders, as reduction factor changed from 0 to $40 \%$. At any reduction factor, the measured pressure is always greater on rigid cylinder. Theoretical pressure curve based on Laplace's law matches fairly well with the pressure measured on rigid cylinder but not so on foam covered cylinder.

As the elastic fabric is placed on soft PVC cylinder, it compresses the foam. Part of the stored energy within the fabric, deforms the foam and dissipates its energy. The same does not happen on rigid cylinder. As a result, the equilibrium for soft cylinder is established at lesser interfacial pressure than that is observed on rigid cylinder surface.

\section{Comparison between measured pressure and estima- ted by energy principle}

The initial $\left(\varepsilon_{0}\right)$ and final elongation $\left(\varepsilon_{l}\right)$ of the fabric corresponding different reduction factor is shown in Table 2. Final elongation is always less than initial elongation. The energy $\left(E_{F a b}\right)$ released by the fabric due to contraction was calculated by integrating Equation 17 for various values of $\varepsilon_{0}$ and $\varepsilon_{1}$ (Table 3 ). By incorporating the values of $\alpha$ and $\beta$, Equation 16 turns to

$$
E_{\text {Foam }}=-4 \times 10^{-7} \frac{p^{3}}{3}+0.0008 \frac{p^{2}}{2}
$$

Now equating Equation 19 to various $E_{F a b}$ values, p was estimated. The theoretical pressures based on Laplace equation and energy consideration along with measured pressure are shown in Table 4. The predicted values based on energy consideration are closer to the measured values where as the values estimated by Laplace equation is not so close.

Table 2 Reduction factor and fabric elongation

\begin{tabular}{lllllll}
\hline $\begin{array}{l}\mathbf{S} \\
\text { No. }\end{array}$ & $\mathbf{R}_{\mathbf{F}}(\%)$ & $\begin{array}{l}\text { Fabric tube } \\
\text { diameter }(\mathbf{c m})\end{array}$ & $\begin{array}{l}\text { Initial diameter of } \\
\text { cylinder }(\mathbf{c m})\end{array}$ & $\begin{array}{l}\text { Initial fabric } \\
\text { elongation }\left(\varepsilon_{0}\right)(\mathbf{c m})\end{array}$ & $\begin{array}{l}\text { Cylinder diameter after } \\
\text { placing fabric tube }(\mathbf{c m})\end{array}$ & $\begin{array}{l}\text { Final fabric } \\
\text { elongation }\left(\varepsilon_{1}\right)(\mathbf{c m})\end{array}$ \\
\hline 1 & 0 & 8.8 & & 0 & 8.8 & 0 \\
2 & 10 & 7.8 & & 1 & 8.6 & 0.8 \\
3 & 20 & 7 & 8.8 & 1.8 & 8.5 & 1.5 \\
4 & 30 & 6.2 & & 2.6 & 8.4 & 2.2 \\
5 & 40 & 5.2 & & 3.6 & 8.2 & 3 \\
\hline
\end{tabular}

Table 3 Energy released by fabric tube

\begin{tabular}{lllll}
\hline S No & $\mathbf{R}_{\mathbf{F}}(\%)$ & Initial fabric elongation $\left(\varepsilon_{0}\right)(\mathbf{c m})$ & Final fabric elongation $\left(\varepsilon_{1}\right)(\mathbf{c m}) \begin{array}{l}\text { Energy released }\left(\mathrm{E}_{\mathrm{Fab}}\right)(\mathrm{gf}- \\
\mathbf{c m})\end{array}$ \\
\hline $\mathrm{I}$ & 0 & 0 & 0 & 0 \\
2 & 10 & 1 & 0.8 & 2.95 \\
3 & 20 & 1.8 & 1.5 & 5.1 \\
4 & 30 & 2.6 & 2.2 & 7.68 \\
5 & 40 & 3.6 & 3 & 13.1 \\
\hline
\end{tabular}

Table 4 Actual and predicted pressure

\begin{tabular}{lllll}
\hline S No & $\mathbf{R}_{\mathbf{F}}(\%)$ & \multicolumn{1}{c}{ Pressure $(\mathbf{m m H g})$} & Predicted & $\begin{array}{l}\text { Actual } \\
\text { measured }\end{array}$ \\
\cline { 3 - 5 } & & \multicolumn{2}{c}{ According to energy consideration } \\
\cline { 3 - 5 } & & According to laplace equation & 0 & 0 \\
\hline 1 & 0 & 0 & 9.7 & 9 \\
2 & 10 & 13.5 & 17.7 & 17 \\
3 & 20 & 25.3 & 25.7 & 24 \\
\hline & 30 & 30.4 & 35.2 & 33 \\
\hline
\end{tabular}




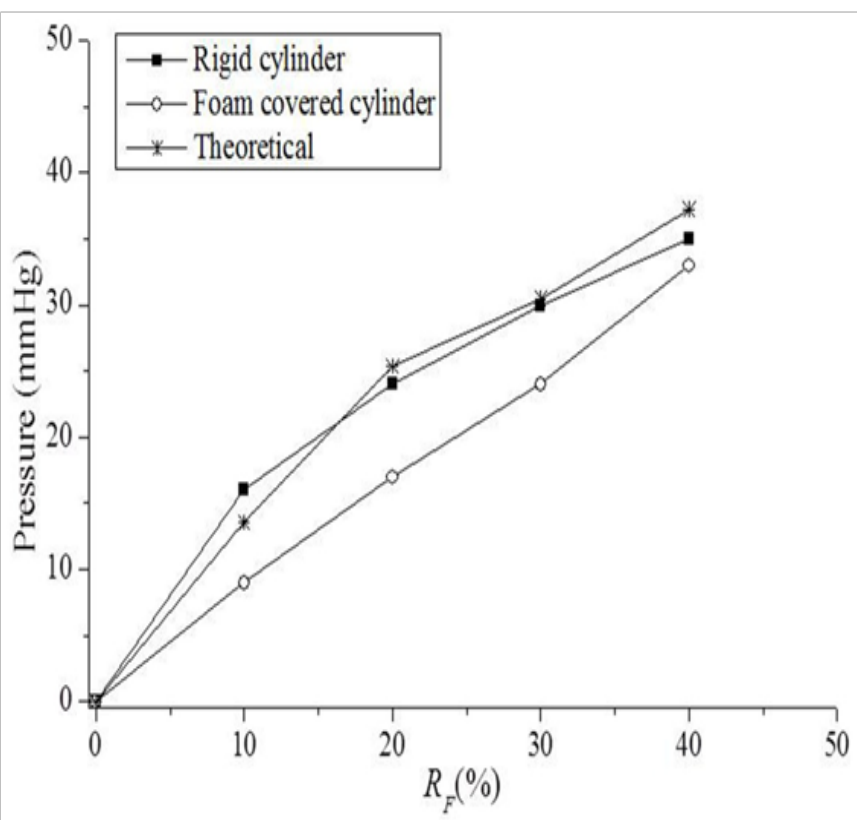

Figure 6 Measured pressure on rigid and foam covered cylinder.

\section{Conclusion}

Pressure generation at the interface of a fabric tube and foam covered cylinder was always less than that on a similar diameter rigid cylinder. Laplace's law was found to estimate pressure on rigid cylinder more accurately than that on soft compressible cylindrical surface. On compressable surface, it over estimates the pressure. Prediction based on energy consideration was found to be closer to actual measured values. Thus energy principle approach could be a used for predicting pressure on human bodies by pressure garments.

\section{Acknowledgments}

None.

\section{Conflict of interest}

Author declares there is no conflict of interest in publishing the article.

\section{References}

1. Giele HF, Liddiard K, Currie K, et al. Direct measurement of catenous pressures generated by pressure garments. Burns. 1997;23(2):137-141.

2. Sawada Y. Pressure developed under pressure garment. Br J Plast Surg. 1993;46(6):538-541.

3. Engrav LH, Heimbach DM, Rivara FP, et al. 12Year within-wound study of the effectiveness of custom pressure garment therapy. Burns. 2010;36(7):975-983.

4. Trenell MI, Rooney KB, Sue CM, et al. Compression garments and recovery from eccentric exercise: A 31P-MRS study. J Sports Sci Med. 2006;5(1):106-114.

5. Troynikov O, Ashayeri E, Burton M, et al. Factors influencing the effectiveness of compression garments used in sports. Procedia Engineering. 2010;2:2823-2829.

6. Thomas $\mathrm{S}$. The use of the Laplace equation in the calculation of sub-bandage pressure. EWMA J. 2003;3(1):21-23.

7. Kirk W, Ibrahim SM. Fundamental relationship of fabric extensibility to anthropometric requirements and garment performance. Textile Research J. 1966;36(1):37-47.

8. Seo H, Kim SJ, Cordier F, et al. Validating a cloth simulator for measuring tight-fit clothing pressure. Proc. SPM 07 Proceedings of the 2007 ACM symposium on Solid and physical modeling. Beijing, China; 2007. p. $431-438$.

9. Lee YJ. Prediction of the clothing pressure based on the 3D shape deformation and mechanical properties of fabrics. $\mathrm{PhD}$ thesis, Depatment of Clothing \& Textiles, Chungnam National University, South Korea; 2005.

10. Hui CL, Ng SF. Model to Predict Interfacial Pressures in Multilayer Elastic Fabric Tubes. Textile Research J. 2001;71(8):683-687.

11. Macintyre L. Designing pressure garments capable of exerting specific pressures on limbs. Burns. 2007;33(5):579-586.

12. Yildiz N. A novel technique to determine pressure in pressure garments for hypertrophic burn scars and comfort properties. Burns. 2007;33(1):59-64.

13. Lihuan Zhao, Xiaohuan Li, Jie Yu, et al. Compression sleeves design based on laplace laws. J Textile Engineering \& fashion technology. 2017;2(2):1-7. 